\title{
REGULATION OF CORONAVIRUS RNA TRANSCRIPTION IS LIKELY MEDIATED BY PROTEIN-RNA INTERACTIONS
}

\author{
X M Zhang ${ }^{1}$ and M M C Lai ${ }^{123}$ \\ ${ }^{1}$ Department of Neurology \\ ${ }^{2}$ Howard Hughes Medical Institute \\ ${ }^{3}$ Department of Microbiology \\ University of Southern California School of Medicine \\ Los Angeles, Calıfornıa
}

\begin{abstract}
Coronavirus mRNA transcription was thought to be regulated by the interaction between the leader RNA and the intergenic (IG) sequence, probably involving direct RNA-RNA interactions between complementary sequences In this study, we found that a 9-nucleotide sequence immediately downstream of the leader RNA up-regulated mRNA transcription and that a particular strain of mouse hepatitıs virus (MHV) lackıng this 9-nucleotıde transcribed subgenomic mRNA species containing unusually heterogeneous leader-fusion sites These results suggest that the sequence complementarity between the leader and IG is not necessarily required for mRNA transcription UV cross-linkıng experıments using cytoplasmic extracts of uninfected cells and the IG sequence showed that three different cellular proteins bound to IG of the template RNA Deletion analyses and site-directed mutagenesis of IG further demonstrated a correlation between protein-binding and transcription efficiency, suggesting that these RNA-binding proteins are involved in the regulation of coronavirus mRNA transcription We propose that coronavirus transcription is regulated by RNA-protein and protein-protein interactions
\end{abstract}

\section{INTRODUCTION}

Coronavirus mRNA synthesis involves a unique mechanism of discontinuous transcription, generating subgenomic mRNAs which contain a leader RNA fused to a distant RNA sequence ${ }^{4}$ The precise mechanism of this RNA synthesis is not yet fully 
understood. Regardless of the mechanism of mRNA transcription, the regulation of coronavirus mRNA synthesis has to involve the interaction between the leader RNA, which is present at the $5^{\prime}$-end of the genomic RNA and is incorporated into subgenomic mRNAs, and the intergenic (IG) sequence, which precedes every gene ${ }^{5}$. In this study, we use mouse hepatitis virus (MHV) as a model system to study the mechanism of the transcriptional regulation. The 3'-end of the leader on MHV genomic RNA generally contains 2 to 4 copies of a pentanucleotide sequence (UCUAA) ${ }^{8}$. The IG sequence also contains a consensus UCUAAAC or similar sequence ${ }^{10}$. The UCUAAAC sequence is necessary and sufficient for the initiation of mRNA synthesis ${ }^{3}$. Fusion of the leader sequence with the mRNA body sequence usually occurs between the UCUAA repeats of the leader and the consensus IG sequence ${ }^{9}$. It has thus been hypothesized that mRNA initiation is mediated by direct RNA-RNA interaction between the leader RNA and consensus $\mathrm{IG}^{4}$. The copy number of UCUAA at the $3^{\prime}$ end of the subgenomic mRNA leader may differ from that of the genomic RNA leader, presumably because of imprecise alignment between the leader and the intergenic regions ${ }^{4,9}$. The $5^{\prime}$-end of genomic RNA, including the leader, also acts as an enhancer-like element for mRNA synthesis ${ }^{6}$. Furthermore, the sequence of IG significantly affects the pattern of transcriptional regulation. For instance, the efficiency of transcription initiation from the IG of mRNA2-1, varies with the leader RNA containing either 2 or 3 UCUAA copies ${ }^{10}$, while the IG of mRNA7 is constitutively transcribed. In addition, a 9-nucleotide (nt) sequence (UUUAUAAAC) immediately downstream of the leader influenced the frequency of incorporation of the leader RNA into mRNAs ${ }^{13}$. Deletion of this sequence also prevented leader-switching during RNA replication ${ }^{8}$. Thus, coronavirus mRNA transcription is regulated by direct or indirect interactions of multiple cis- and trans-acting RNA sequences.

In this study, we found that a particular strain of MHV, JHM2c, which has a deletion of the 9-nt sequence immediately downstream of the leader, generated subgenomic mRNAs with very heterogeneous leader-fusion, which did not directly involve complementary RNA sequences. These results strongly suggest that leader-mRNA fusion in coronavirus transcription does not require direct RNA-RNA interaction between complementary sequences. Ultraviolet (UV) light cross-linking studies with cytoplasmic extracts further identified several cellular proteins binding to the IG sequence and/or the leader RNA, both being the RNA regulatory elements. Furthermore, site-directed mutagenesis of IG7 demonstrated a correlation between the protein-binding and transcription efficiencies. This study thus provides the first evidence that cellular RNA-binding proteins are involved in coronavirus RNA synthesis, suggesting that these RNA-binding proteins may be the transcription factors.

\section{MATERIALS AND METHODS}

Viruses and cells: MHV strains JHM(3) and JHM2c(3) contain three UCUAA repeats, and JHM2c(4) contains four UCUAA repeats at the 3 '-end of the leader RNA. Both JHM2c(4) and JHM2c(3) have a deletion of a 9-nt sequence immediately downstream of the leader. The properties of these viruses have been described previously ${ }^{10}$. DBT cells ${ }^{2}$ were used for infections and transfections.

$U V$ cross-linking of RNA-protein complex: Cytoplasmic extracts were prepared from uninfected DBT cells and UV cross-linking experiments were performed as described previously'. 


\section{RESULTS AND DISCUSSION}

\section{Effects of the Nine-Nucleotide Sequence Immediately Downstream of the Leader RNA on mRNA Transcription}

By using an MHV DI RNA containing a CAT gene behind an IG, we have previously demonstrated that deletion of the 9-nt sequence (UUUAUAAAC) in DI RNA reduced the efficiency of transcription from the IG, suggesting the involvement of this sequence in the regulation of subgenomic mRNA transcription ${ }^{13}$. Furthermore, the deletion of the 9-nt sequence inhibited the ability of the leader RNA of the DI RNA to be incorporated into subgenomic mRNAs, suggesting that this sequence might be required as a transcriptional terminator to synthesize a free leader RNA ${ }^{13}$. Thus, an intriguing question arises: if an MHV genome (e.g. JHM2c) lacks this 9-nt sequence, how are viral subgenomic mRNAs transcribed? To answer this question, we constructed several DI RNA-reporter plasmids which contain a CAT gene behind an IG of mRNA7 or mRNA2-1 $1^{6,13}$, in the presence or absence of the 9-nt sequence immediately downstream of the leader (Fig.1). These RNAs were transfected into cells infected with JHM2c, which lacks the 9-nt sequence in the genomic RNA. The transcriptional efficiencies from each IG site were determined by CAT assays, which accurately reflect transcriptional efficiencies ${ }^{6}$. As shown in Table 1, in the presence of JHM2c(3) and JHM2c(4), both of which lack the 9-nt sequence, CAT activities were approximately 3 -fold higher from DIs with the 9-nt sequence than those from DIs without the 9-nt sequence. For example, in JHM2c(3)-infected cells, CAT activity was 3-fold higher in 25CAT+9nt than 25CAT, and 7-fold higher in DECAT2-1+9nt than DECAT2-1. Similarly, in JHM2c(4)-infected cells, CAT activity of DECAT-2-1+9nt was approximately 3.5-fold higher than in DECAT2-1. DECAT2-1(2)m RNA, which is identical to DECAT2$1+9$ nt except that it contains only 2 UCUAA repeats (Fig.1), has a transcriptional efficiency similar to that of DECAT2-1+9nt in both infected cells, suggesting that the differences in transcriptional efficiencies among different DI constructs were mainly due to the presence or absence of the 9-nt sequence, and not the copy number of UCUAA or other differences within the leader sequence. However, in JHM2c(4)-infected cells, 25CAT and 25CAT+9nt exhibited similar transcriptional efficiencies. These results indicate that the 9-nt sequence in cis (i.e. in DI RNA) could affect CAT expression, depending on the context of the IG and helper virus. This result is consistent with the previous finding that MHV transcription is regulated by both trans- and cis-acting leaders and the IG sequence ${ }^{13}$. Nevertheless, subgenomic mRNAs were transcribed from almost all of the DI RNA constructs, even though both the helper virus and DI RNA lack the 9-nt sequence. Therefore, we conclude that this 9-nt sequence is not absolutely necessary for transcription. When $\operatorname{JHM}(3)$, which contains the 9-nt sequence, was used as a helper virus, CAT activities in all of the RNA constructs were generally higher than in the corresponding RNAs in JHM2c(3)-infected cells (Table 1). These results together suggest that the 9-nt sequence in either helper viral RNAs (in trans) or DI RNA (in cis) could enhance transcription of subgenomic mRNAs. However, the magnitude of these effects varied with respect to the context of other

Figure 1. Structures of the cDNA clones of DI RNAs. Only the DI-derived regions, which are placed behind a T7 RNA polymerase promoter, are shown. XbaI was used for digestion of constructions for in vitro run-off transcription. Each unlabeled small open rectangle in the leader and IG represents a copy of UCUAA. The V-shaped breaks in the lines represent a 9-nucleotide deletion.

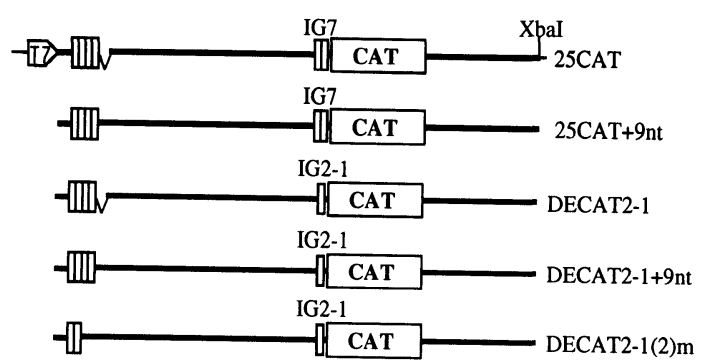


Table 1. Effect of the nine-nucleotide sequence on CAT expression

\begin{tabular}{lccc}
\hline & \multicolumn{3}{c}{ CAT activity (fold increase) } \\
\cline { 2 - 4 } Transfected RNA & JHM2c(3) & JHM2c(4) & JHM(3) \\
\hline 25CAT & $67 \times$ & $252 \times$ & $291 \times$ \\
25CAT+9nt & $200 \times$ & $225 \times$ & $277 \times$ \\
DECAT2-1 & $1 \times$ & $7 \times$ & $11 \times$ \\
DECAT2-1+9nt & $7 \times$ & $24 \times$ & $36 \times$ \\
DECAT2-1(2)m & $6 \times$ & $19 \times$ & $70 \times$ \\
\hline
\end{tabular}

*The data represent the averages of five separate infection and transfection experiments in DBT cells. CAT activity is indicated as fold increase against the background values for a culture in which either virus for infection or RNA for transfection alone was used as a negative control, which was set at $1 \times$.

sequences, such as the number of UCUAA repeats in the leader and IG (either IG7 or IG2-1) in both the DI RNA and helper virus. Since the 9-nt sequence is not part of the leader RNA and is not incorporated into mRNAs, this result further indicates that these are cis-acting upstream regulatory sequences for mRNA transcription.

\section{Heterogeneity of Leader-mRNA Fusion Sites}

We further determined the structure of subgenomic mRNAs from cells infected with JHM2c(3), and transfected with 25CAT, 25CAT+9nt or DECAT2-1(2)m RNA by sequencing. We found that, when JHM2c(3), which lacks the 9-nt sequence, was used as a helper virus, the leader-mRNA fusion sites in subgenomic mRNAs were extremely heterogeneous. Similar heterogeneity was also detected in mRNA2-1 of JHM2c(3)-infected cells ${ }^{12}$. Most notably, in 10 of 53 clones sequenced, the leader is fused with mRNAs at a site either upstream or downstream of the consensus IG. These sites bear little or no sequence homology with the leader. As a result, mRNAs contain sequences upstream, or have a sequence deletion downstream, of the consensus UCUAAAC. These heterogeneous leader-mRNA fusions suggest that coronavirus mRNA transcription does not require a complementary sequence between the leader and IG, implying that the RNA regulatory elements must be brought together by other factors such as cellular proteins which bind to these RNAs.

\section{Interactions between the Cytoplasmic Proteins and the Intergenic Sequence of MHV RNA}

We have previously shown that some cellular proteins, most notably $\mathrm{p} 35 / 38$, bind to the template strand of the leader RNA. Another protein $\mathrm{p} 55$ binds to the leader RNA per se ${ }^{1}$. To determine whether any cellular proteins interact with the third component of the RNA regulatory sequences, i.e. IG sequence, of MHV RNA, we performed UV cross-linking experiments using cytoplasmic extracts of uninfected DBT cells and the template strand of IG7. We found that four major proteins p110, p70, p48, and p35/38 bound to IG7-SF135(-) RNA. To determine the binding specificity of these proteins, we used an unlabeled homologous RNA and a nonspecific vector RNA to perform competition assays. When increasing amounts of the unlabeled homologous RNA (2- to 50-fold molar excess) were preincubated with a fixed amount of cytoplasmic extracts ( $20 \mu \mathrm{g}$ of cellular proteins), most of the protein bands were competed away, except that p110 was not efficiently competed. In contrast, the 
nonspecific vector sequence (25- to 50 -fold molar excess) did not affect the amount of protein-binding(data not shown) We conclude that at least three cytoplasmic proteins (p70, p48, and p35/38) specifically bound to IG7-SF 135(-) RNA, while p110 binding was probably not specific Using a series of deletion constructs, we found that $\mathrm{p} 35 / 38$ bound only to the fragments which contain the consensus sequence of IG7, but not RNAs without the consensus sequence We thus conclude that the consensus sequence of IG7 is essential for $\mathrm{p} 35 / 38$ binding In contrast, the binding of p70 and p48 was only slightly decreased in some of the deletion clones, suggesting that the sequence requirement for $\mathrm{p} 70$ and $\mathrm{p} 48$ was not very specific or that these two proteins bound to a longer stretch of RNA sequence

\section{Site-Specific Mutagenesis of IG7 Reveals That RNA-Protein Interaction Correlated with the Transcription Efficiency of Subgenomic mRNAs}

To test the hypothesis that cellular proteins may be involved in the regulation of mRNA transcription, we constructed five different IG mutants (Fig 2A), which have previously been shown to result in altered transcriptional efficiencies ${ }^{37}$, for UV cross-linkıng studies with cytoplasmic extracts The results showed that the binding efficiencies of these IG mutants with p35/38 correlated approximately with their transcriptional efficiencies,

A.

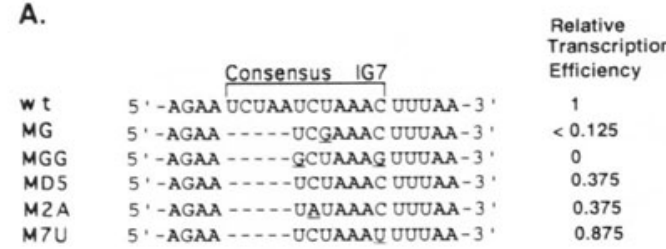

B.

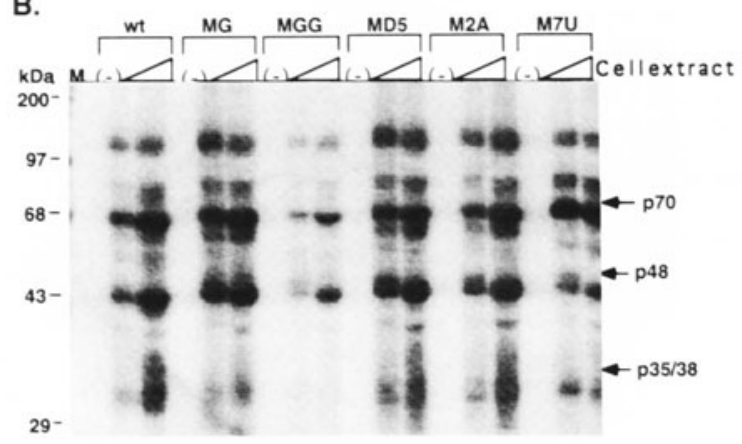

Figure 2. Site-specific mutagenesis of the consensus sequence of IG7 with regards to their protein binding properties (A) Sequences (in positive sense) of the wild-type (wt) IG7 and the mutants used The consensus sequence of the wt IG7 is indicated at the top of the sequence Nucleotide mutations are underlined, and deletions are indicated by dashes The transcription efficiencies of the mutant IGs indicated are relative to that of the wt IG, which is set as $100 \%$ These data are recalculated from the published reports ${ }^{37}$ (B) UV cross-linkıng of cellular proteıns with negative strand RNAs of the wt and mutant IG7 Cytoplasmic extracts ( 2 and $20 \mu \mathrm{g}$ proteins, respectively) were mixed with a fixed amount of ${ }^{32} \mathrm{P}$-labeled RNAs and UV-irradiated In (-) lanes, no cell extract was added $M$ molecular mass standard in kDa Proteın bands p70 p48 and p35/38 are indicated with arrows on the right side 
except for mutant M7U (Fig. 2A and B). Specifically, mutant MGG, which has a deletion of a UCUAA sequence and two additional single-nucleotide mutations, has been shown to lack the transcriptional activity. Correspondingly, MGG(-) RNA did not bind p35/38, and bound slightly less p48 and p70. Mutant MG, which has a deletion of a UCUAA sequence and a C-_G mutation, had retained less than $12.5 \%$ of transcription efficiency of the wild-type IG7. Correspondingly, this IG mutant bound significantly less p35/38 than the wild-type RNA. Mutants M2A and M7U, which had similar transcription efficiencies, also had similar binding activities with $\mathrm{p} 35 / 38$. These results demonstrated that the binding efficiencies of p35/38 to IG7 correlated roughly with the transcription efficiencies of these mutants, suggestıng that their binding to IG7 is required for subgenomic mRNA transcription, Interestingly, a $\mathrm{C}->\mathrm{U}$ mutation in mutant $\mathrm{M} 7 \mathrm{U}$, which had retained most of the transcription activity, bound a relatively small amount of $\mathrm{p} 35 / 38$, but it bound an increased amount of $\mathrm{p} 70$ (Fig. 2). Thus, $\mathrm{p} 70$ binding may substitute for $\mathrm{p} 35 / 38$ in restoring the transcription activity. These results suggest that these cellular RNA-binding proteins may be transcription-associated factors for coronavirus RNA synthesis.

Recently, we have suggested that protein-RNA and protein-protein interactions rather than direct RNA-RNA interactions are the driving force for the initiation of coronavirus RNA transcription ${ }^{13}$. This model proposes that viral and cellular proteins first bind to the RNA regulatory elements through protein-RNA interactions; these components are then brought together with viral RNA polymerase and other viral and cellular factors to form a transcription initiation complex through protein-protein interactions (Fig.3). In this model, the sequence complementarity between leader and IG is not absolutely required; they may only

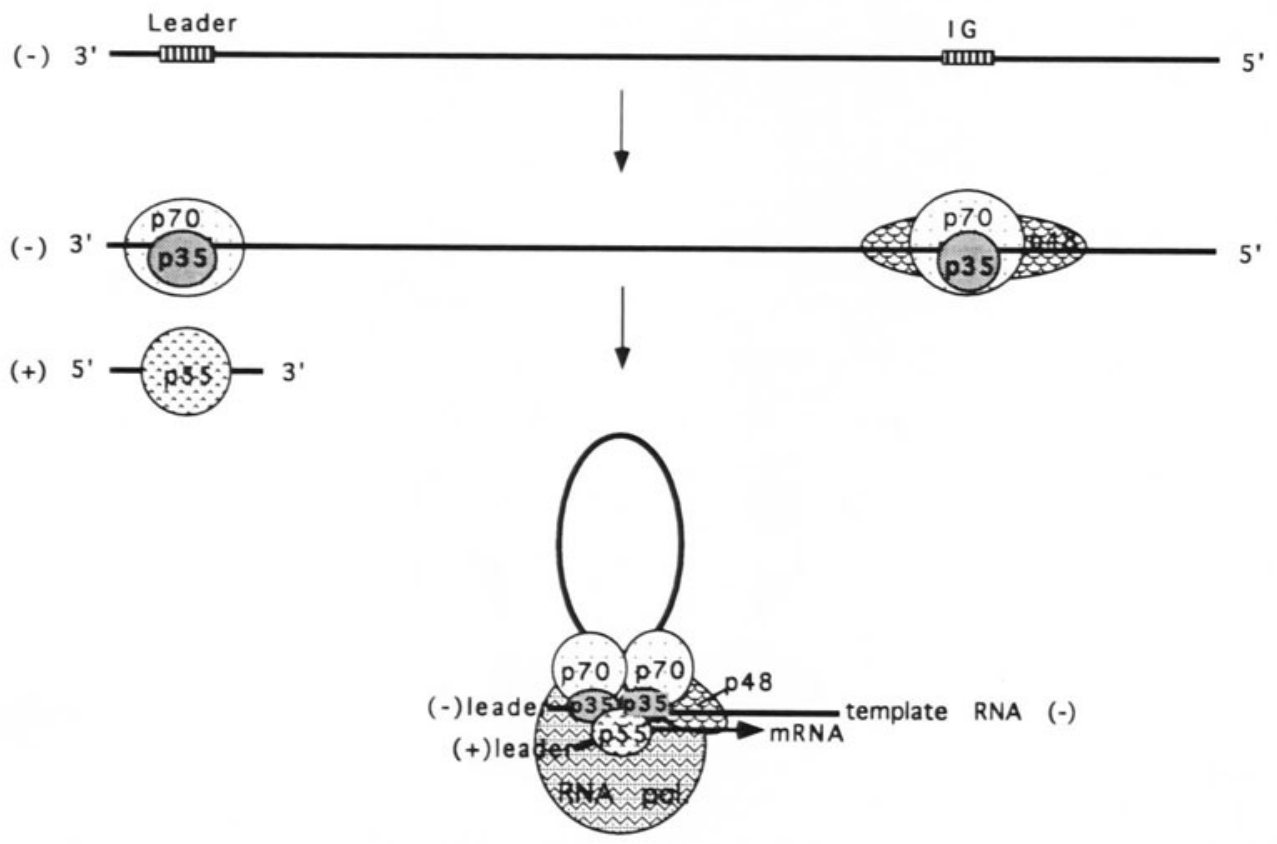

Figure 3. Model of coronavirus RNA transcription through protein-RNA and protein-protein interactions The striped boxes indicate the UCUAA repeats at the 3 '-end of the leader and the consensus IG The proteins bound to the different regions were identified in reference ${ }^{1}$ and this study The presence of the putative viral RNA polymerase in the complex is hypothetical In this putative complex, the negative-strand, genome-length RNA serves as a template, and the leader RNA (positive sense) in trans binds to the negative-strand IG RNA and serves as a primer The subgenomic mRNA transcription starts at the IG region inside the complex 
provide the recognition signals for viral and cellular proteins Any alterations of the RNA elements and protein components within this complex could possibly affect the initiation and efficiency of subgenomic mRNA transcription The identification of the unusual heterogeneity in leader-mRNA fusion and cellular RNA-binding proteins involving in transcriptional regulation in this study strongly supports this notion

\section{ACKNOWLEDGMENTS}

This work was supported by a Public Health Service Research Grant AI16144 from the National Institutes of Health M M C L is an Investigator of the Howard Hughes Medical Institute

\section{REFERENCES}

1 Furuya T, Lai M M C Three different cellular proteins bind to complementary sites on the $5^{\prime}$-end-positive and 3'-end-negative strands of mouse hepatitıs virus RNA J Virol 1993,67 7215-7222

2 Hırano N , Fujıwara K, Hino S, Matsumoto M Replication and plaque formation of mouse hepatitis virus (MHV-2) in mouse cell line DBT culture Arch Gesamte Virusforsch 1974,44 298-302

3 Joo M, Makıno S Mutagenic analysis of the coronavirus intergenic consensus sequence J Virol 1992,66 6330-6337

4 Laı M M C Coronavirus organızation, replication and expression of genome Annu Rev Microbiol 1990,44 303-333

5 Lai M M C , Barıc R S, Brayton P R, Stohlman S A Characterization of leader RNA sequences on the virion and mRNAs of mouse hepatitis virus, a cytoplasmic RNA virus Proc Natl Acad Scı USA 1984,81 3626-3630

6 Liao C L, La1 M M C Requirement of the 5'-end genomic sequence as an upstream $c l s$-actıng element for coronavirus subgenomic mRNA transcription J Virol 1994,68 4727-4737

7 Makıno S, Joo M Effect of intergenıc consensus sequence flankıng sequences on coronavirus transcrıption J Virol 1993,67 3304-3311

8 Makıno, S , Laı M M C High-frequency leader sequence switchıng durıng coronavirus defectıve interferıng RNA replication J Virol 1989,63 5285-5292

9 Makıno S, Soe L H, Shıeh C K, La1 M M C Discontınuous transcription generates heterogeneity at the leader fusion sites of coronavirus mRNAs J Virol 1988,62 3870-3873

10 Shieh C K, Lee H J, Yokomorı K, La Monıca N, Makıno S, La1 M M C Identification of a new transcriptional initiation site and the corresponding functional gene $2 \mathrm{~b}$ in the murine coronavirus RNA genome J Virol 1989,63 3729-3736

11 Shieh C K, Soe L H, Makıno S, Chang M F, Stohlman S A, La1 M M C The 5'-end sequence of the murine coronavirus genome implications for multiple fusion sites in leader-primed transcription Virology 1987,156 321-330

12 Zhang X M, Lai M MC Unusual heterogeneity of leader-mRNA fusion in a murine coronavirus Implications for the mechanism of RNA transcription and recombination J Virol (in press)

13 Zhang X M, Liao C L , Lai M M C Coronavirus leader RNA regulates and initiates subgenomıc mRNA transcription both in trans and in cls J Virol 1994,68 4738-4746 